\title{
Kernos
}

Revue internationale et pluridisciplinaire de religion grecque antique

6 | 1993

Varia

\section{Platon et la sagesse de l'Égypte}

James McEvoy

URL : http://journals.openedition.org/kernos/550

DOI : $10.4000 /$ kernos.550

ISSN : 2034-7871

Éditeur

Centre international d'étude de la religion grecque antique

Édition imprimée

Date de publication : 1 janvier 1993

Pagination : 245-275

ISSN : 0776-3824

Référence électronique

James McEvoy, "Platon et la sagesse de l'Égypte », Kernos [En ligne], 6 | 1993, mis en ligne le 07 avril 2011, consulté le 19 avril 2019. URL : http://journals.openedition.org/kernos/550 ; DOI : 10.4000/ kernos.550

Kernos 
Kernos, 6 (1993), p. 245-275.

\section{PLATON ET LA SAGESSE DE LÉGYPTE ${ }^{1}$}

On trouve dans l'antiquité une tradition, largement répandue, selon laquelle Platon aurait visité l'Égypte. Strabon, qui est un des plus anciens témoins de cette tradition, associe le nom de l'astronome Eudoxe de Cnide à la visite de Platon ${ }^{2}$, et il ajoute qu'Eudoxe, qui était l'élève et l'ami du fondateur de l'Académie, utilisa un observatoire égyptien et composa un livre dans la tradition d'Hécatée, d'Hérodote et de Ctésias. De larges fragments de ce livre étaient relatifs à l'Égypte et traitaient des inondations du Nil, des règles des prêtres d'Héliopolis, d'une des tombes supposées d'Osiris et du mythe d'Ammon ${ }^{3}$. Cicéron, qui est le premier à en parler, prétend que Platon se rendit deux fois en Égypte, avant ses voyages en Italie et en Sicile, mais nous ignorons la source de son information. Diogène Laërce, pour sa part, affirme que notre philosophe se déplaça à Cyrène, dans le but de visiter son ami Théodore (un des personnages du Politique), ce qu'on trouve également chez Apulée. Ensuite, poursuit Diogène, Platon gagna l'Italie pour faire la connaissance des Pythagoriciens, et de là il se rendit en Égypte "pour y rencontrer les prophètes» (mantines). Ammien Marcellin reprend lui aussi l'histoire du voyage qu'auraient effectué en Égypte aussi bien Solon que Platon ${ }^{4}$. De même, dans les derniers temps de l'empire

1 Cet article est la version remaniée d'une contribution parue en anglais dans l'Irish Philosophical Journal, vol. I, $\mathrm{n}^{\circ} 2$ (autumn 1984), sous le titre «Plato and the Wisdom of Egypt». Je tiens ici à remercier deux lecteurs qui ont eu l'amabilité de me communiquer leurs remarques et des références supplémentaires à des écrits anciens et modernes : M.G.L. Huxley, professeur émérite à l'Université de Belfast, et M.A.F. Shore, professeur d'égyptologie à l'Université de Liverpool. Qu'ils trouvent ici l'expression de toute ma gratitude. Mes remerciements vont aussi à mon assistant, M. Jacques Follon, qui a traduit cet article en français, ainsi qu'à mon collègue, M. Olivier Depré, qui m'a aidé dans la correction des épreuves.

2 W.K.C. GuThrie, A History of Greek Philosophy, vol. IV, p. 14.

3 Ibid., vol. V, p. 451 et 456.

4 Pour les références des traditions anciennes relatant un voyage de Platon en Égypte, voir H. LeISEgANG, art. Platon, in $R E$, 2. Reihe, XI. Halbb. (1941), col. 2350; J. KeRsChENSTEINER, Platon und der Orient, Stuttgart, 1945. Dans la liste des auteurs anciens, Kerschensteiner inclut VALÈRE MAXIME (Factorum et 
romain, on voit saint Augustin associer plus d'une fois Platon à l'Égypte, puisqu'en plusieurs endroits de son De Civitate Dei, il fait allusion à un voyage du philosophe athénien dans ce pays et met explicitement en rapport cette visite supposée avec la sagesse des Égyptiens et de certains Juifs qui vivaient, selon lui, parmi eux ${ }^{5}$.

À propos de cette ancienne tradition, les historiens et les philologues modernes sont fort partagés. En effet, beaucoup d'entre eux ne cachent pas leur scepticisme, en raison de l'absence de tout témoignage antérieur et digne de foi concernant un tel voyage de Platon, et aussi à cause du récit autobiographique de la Septième Lettre, où l'auteur nous donne une information abondante et précieuse sur ses deux voyages en Sicile, mais reste silencieux à propos de l'Égypte ${ }^{6}$. D'emblée, je dois dire que je ne puis fournir aucune information nouvelle sur cette question et d'ailleurs je tiens à souligner que je ne cherche nullement à prouver que Platon a fait un voyage en Égypte. Mon propos est plutôt de montrer quelle connaissance il avait de ce pays fascinant et comment il a mis cette connaissance au service de ses idéaux philosophiques. Je m'attacherai donc aux références à l'Égypte qu'on peut trouver dans le Timée et les Lois, ainsi qu'à la sagesse que Platon a amassée et incorporée dans ces deux écrits de sa vieillesse. En rapport avec ce matériau égyptien qu'on trouve dans le Timée et les Lois, j'avancerai également

Dictorum Memorabilium, VIII, 7, 3), Diodore DE SicIle (Bibliotheca, I, 96) et Clément d'Alexandrie (Protrepticus, VI, 70).

5 S. Augustin, De Civitate Dei, VIII, 9; sur Platon et les Juifs en Égypte voir ibid, VIII, 11. Beaucoup de Pères de l'Église croyaient que Platon dépendait des prophètes de l'Ancien Testament, et, à travers eux, de Moïse. Saint Ambroise s'appuie sur les récits du voyage de Platon en Égypte pour mettre le philosophe athénien en rapport avec le prophète Jérémie, qui s'y exila à la suite de l'invasion de la Palestine par les Babyloniens. Saint Augustin a bien vu qu'il y avait là un anachronisme, mais il admet la possibilité que la religion juive ait pu exercer une influence sur le fondateur de l'Académie. Sur tout cela, voir l'état de la question bien documenté de Paul Cinolas, Plato : the Attic Moses? Some Patristic Reactions to Platonic Philosophy, in CW, 2 (1978-1979), p. 217-225.

6 Ainsi, à la suite de Wilamowitz et de Schubart, A.E. Taylor estime que les observations relatives à l'Égypte dans les Lois «semblent être de première main" (Plato. The Man and his Work, p. 4). En revanche, Kerschensteiner conclut son examen des anciens témoignages et des opinons modernes sur une note sceptique : «So wird schon durch das Schwanken der Überlieferung auch die ägyptische Reise als sehr unwahrscheinlich erwiesen und in eine Reihe gerückt mit den Nachrichten von den anderen Reisen, die sich um den festen Kern der sizilischen Reise ansetzten und allmählich fortwucherten, bis man schließlich Platon die ganze oikoumene bereisen ließ» (op. cit., p. 50). 
certaines idées concernant la forme prise par la philosophie de Platon dans les dernières années de sa vie. Mais le mieux est peut-être de commencer par un bref résumé du mythe de l'Atlantide (Timée 21a26d), avant de fournir un commentaire de cette histoire dans son rapport avec l'Égypte7.

\section{Le mythe de l'Atlantide}

Voyons tout d'abord le cadre du mythe d'Athènes et de l'Atlantide. Dans le dialogue fictif qui ouvre le Timée, Platon fait dire à Socrate que, la veille de sa rencontre avec le dénommé Timée de Locres, il a tenu un discours sur "la constitution la plus parfaite et par quels hommes elle doit être appliquée» (17c). C'est là, très probablement, une allusion à la République. Car les interlocuteurs de Socrate dans le Timée, à savoir Timée lui-même, Critias et Hermocrate, étaient déjà les siens dans la République. Et ils expriment ici leur adhésion aux institutions qui ont été décrites dans ce dernier dialogue, à savoir la séparation des artisans et des guerriers, l'austère éducation idéale et la vie en commun imposées à ces derniers, l'éducation des femmes et la procréation planifiée des enfants... Mais maintenant Socrate souhaite entendre décrire la manière dont cette construction idéale pourrait être réalisée dans le monde de l'action, de la guerre et de la diplomatie (19b-c). S'estimant lui-même incapable de cette description, il appelle à la rescousse ses amis, qu'il juge plus qualifiés : Timée, Critias d'Athènes et Hermocrate de Sicile. Critias est ainsi prié de répéter une histoire qu'il a déjà racontée à ses deux amis la veille au soir et qui pourrait satisfaire la requête de Socrate. Cette histoire lui est revenue en mémoire en

7 La littérature sur l'Atlantide est immense. Dans sa History of Greek Philosophy (vol. V, p. 247-50), GUTHRIE évoque brièvement les principales théories émises à ce sujet (qui essayent de mettre en rapport le mythe platonicien avec la destruction des îles de Santorin à la suite d'une éruption volcanique, vers 1500 av. J.-C., ou avec les souvenirs de la Crète minoenne), et il renvoie aux travaux (y compris les utopies) que cette histoire a suscités. Le livre de James B RAMWELL, Lost Atlantis (1937), résume les différentes théories. Dans son Zakros (New York, 1971), l'archéologue Nicolas Platon soutient que les souvenirs lointains de la civilisation minoenne, qui peuvent avoir été conservés en Égypte, ont été intégrés par Platon dans son tableau de l'Atlantide. Récemment Wolfgang SCHENKEL (Atlantis : die "namenlose" Insel, in Göttinger Miszellen, 36 [1979], p. 57-60) a même prétendu trouver une étymologie égyptienne au mot Atlantis. Mais il ne faudrait pas oublier que les dialogues platoniciens Timée et Critias restent nos seules sources en ce qui concerne cette histoire. 
entendant le discours de celui-ci sur la république idéale. Il l'a apprise, dit-il, "il y a bien longtemps", de son grand-père Critias, qui l'avait reçue lui-même de Solon, parent et grand ami de son propre père, et elle se rapporte à un antique exploit d'Athènes, qu'il convient de rappeler aujourd'hui "pour rendre à la déesse, à l'occasion de cette fête, un juste et véritable hommage, comme si nous chantions un hymne à sa louange" (21a). L'histoire remonte donc à Solon, le sage législateur, qui était aussi un poète de talent, car, s'il n'en avait pas été empêché par les factions et les luttes politiques qui déchiraient Athènes à son époque, il aurait sûrement réussi à achever la mise en vers du récit qu'il avait luimême rapporté d'Égypte. Solon fit un voyage à Saïs, dans le Delta du $\mathrm{Nil}$, et il interrogea les prêtres de l'endroit sur les antiquités, estimant que "ni lui, ni aucun autre Grec n'en avait pour ainsi dire aucune connaissancen. Un des prêtres se mit à opposer la jeunesse des Grecs à l'âge vénérable de son propre peuple, dont les hauts faits, disait-il, avaient été consignés par écrit dans les temples, où ils étaient conservés depuis des milliers d'années, ayant survécu à des catastrophes naturelles, telles que déluges et tremblements de terre, qui, chez les autres peuples, avaient régulièrement mis fin à toute vie civilisée, rompant ainsi tout lien avec le passé et les obligeant à recommencer chaque fois à zéro. C'est ainsi, poursuivit-il, que l'histoire d'Athènes avant le dernier déluge avait été oubliée par les Athéniens, mais conservée par les Égyptiens grâce à l'antiquité de leurs archives et à leur intérêt pour les anciens Athéniens et leurs descendants.

Neuf mille ans auparavant, c'est-à-dire un millier d'années avant la fondation politique de l'Égypte, Athènes était au faîte de la gloire, grâce à de sages lois et à la noblesse de ses habitants. Sa législation ressemblait fort à celle de l'Égypte à l'époque du récit du prêtre, car elle comportait la séparation des classes et, «à partir des premiers principes qui touchent l'univers», elle avait réglé la découverte des arts utiles à la vie humaine et l'acquisition de toutes les sciences. L'ancienne Athènes avait été dotée par la déesse Athéna, qui «aimait à la fois la guerre et la science», de toutes sortes de qualités, de sorte que les Athéniens d'alors surpassaient «tous les hommes dans tous les genres de mérite, comme on pouvait l'attendre de rejetons et d'élèves des dieux».

La valeur d'Athènes se révéla de la manière la plus éclatante dans sa résistance à l'impérialisme tyrannique de l'Atlantide, une gigantesque puissance insulaire établie au-delà des colonnes d'Hercule. Les rois de cette île tenaient déjà l'Afrique jusqu'aux frontières de l'Égypte, et l'Europe jusqu'à la Tyrrhénie, quand ils entreprirent d'asservir la 
Grèce. Bientôt abandonnée par ses alliés, Athènes combattit seule, ce qui ne l'empêcha pas de remporter la victoire et de rendre à tous les peuples méditerranéens leur liberté. Par après, tremblements de terre et inondations détruisirent l'Atlantide, qui disparut sous les flots, en même temps que la flotte athénienne.

A ce moment de son récit, Critias se tourne vers Socrate et lui dit : "Voilà, Socrate, brièvement résumé, ce que m'a dit Critias [N.B. : le grand-père de l'interlocuteur de Socrate], qui le tenait de Solon. Hier, quand tu parlais de ta république et que tu en dépeignais les citoyens, j'étais émerveillé, en me rappelant ce que je viens de dire. Je me demandais par quel merveilleux hasard tu te rencontrais si à propos sur la plupart des points avec ce que Solon en avait dit» (25e).

En réalité, ce que nous avons là est une évocation très précise de la société égyptienne ${ }^{8}$. Sans doute d'aucuns ont-ils cherché à déterminer le sens du récit, en le posant à l'origine de la pensée utopique (comme Francis Bacon dans sa Nouvelle Atlantide), ou bien en l'interprétant comme une addition, inventée de toutes pièces, au mythe historique d'Athènes défendant vaillamment la liberté des Grecs contre la grande puissance ennemie qu'était la Perse, c'est-à-dire comme quelque chose qui serait en somme un substitut platonicien de l'éloge d'Athènes par Périclès ${ }^{9}$. Mais, à mon avis, dans ce récit Platon n'a pas voulu forger une utopie (en effet, l'Atlantide représente la puissance sans plus, c'està-dire, aux yeux de Platon, un phénomène sans intérêt, tout à l'opposé de l'Idée), ni davantage glorifier l'Athènes de son temps (qu'il considérait comme irrémédiablement perdue au point de vue politique). Aussi aborderai-je ce mythe selon une autre approche. Tout d'abord, je poserai la question historique de savoir ce que Platon savait de l'Égypte et quelle fut la base des informations et des connaissances sur lesquelles il s'appuya pour construire son récit $^{10}$. Ensuite, j'essayerai de déterminer quel est le sens profond du mythe, dans la mesure où il essaye de rattacher l'idéal politique de la République au mythe de l'ordre du monde qu'on trouve dans le Timée. Enfin je m'efforcerai de voir si le mythe en question jette quelque lumière sur l'entreprise platonicienne, dans le

8 Cf. infra, 4 : «L'Égypte et l'Idée platonicienne».

9 Ou même sa réconciliation avec Athènes (voir P. FrIEDLÄNDER, Plato, vol. I : An Introduction).

10 Je précise que je tiens le mythe de la guerre entre Athènes et l'Atlantide pour une pure fiction. 
développement continu qu'elle a suivi, depuis la République jusqu'aux Lois.

\section{Ce que Platon savait de l'Égypte}

Dans la connaissance que Platon avait de l'Égypte, quatre éléments méritent d'être examinés du point de vue de l'«historicité" :

1. La mention, dans le préambule, de l'Égypte, de Saïs, du roi Amasis et de la déesse Neith.

2. La parenté, admise par Platon, entre Athènes et l'Égypte : s'agit-il d'une pure fiction, ou bien est-ce effectivement un trait de la conscience historique qu'Athènes avait d'elle-même ?

3. Le contraste entre la jeunesse de la Grèce et le grand âge du peuple égyptien.

4. Les lois égyptiennes ${ }^{11}$.

\subsection{Platon et Hérodote}

Il est impossible de ne pas remarquer l'aisance avec laquelle Platon parle de l'Égypte, car il le fait en des termes grecs qui sont apparemment bien établis (le Nil, le Delta, le nome [district administratif] de Saiis, la déesse Neith) et qui, à l'exception des noms propres (qui apparaissent sous une forme hellénisée), ne doivent rien à la langue égyptienne. Visiblement, notre auteur était familier de concepts égyptiens qui avaient déjà été transposés dans sa langue maternelle. D'ailleurs, nous autres, modernes, sommes aussi en cette matière les héritiers et les débiteurs des Grecs. Car nous voyons précisément l'Égypte avec des yeux grecs et nous nous servons encore de mots issus du grec pour désigner, de l'Égypte, aussi bien des choses (telles que les pyramides, le delta, les crocodiles, l'obélisque, le papyrus, les hiéroglyphes, le Nil, le Sphinx, pour ne rien dire du nom d'«Égypte» ${ }^{12}$ ), que des cités (Memphis, Thèbes, Héliopolis, Hermopolis, Éléphantine...). Et les noms mêmes des pharaons et des dynasties n'ont survécu que dans le

11 À cette liste, on pourrait encore ajouter la connaissance et la compréhension que Platon avait de l'art égyptien (cf. infra, 4: «L'Égypte et l'Idée platonicienne»).

12 Les Égyptiens utilisaient notamment «Hi-ku-ptah», qui signifie "résidence de l'âme de Ptah", pour désigner Memphis, mais ils n'utilisaient jamais ce terme pour l'ensemble du pays. Voir à ce sujet J. CERNÝ, Language and Writing, in J.R. HARRIS (ed.), The Legacy of Egypt, 2e éd., Oxford, 1971, p. 204. 
grec hellénistique du prêtre égyptien Manéthon ${ }^{13}$. Les seuls noms proprement égyptiens à avoir connu une survie notable dans une langue postérieure semblent être "0asis" et "désert» 14 . Finalement, il est assez normal que nous utilisions encore le vocabulaire de cette époque hellénistique, car celle-ci a fait de l'Égypte une partie intégrante du monde méditerranéen et de l'oikoumenê, où dominait largement la langue grecque. L'ancienneté de l'Égypte, ses hiéroglyphes mystérieux, ses traditions sacerdotales, ses temples fabuleux ainsi que ses richesses et sa sagesse légendaire devinrent dans la littérature hellénistique et romaine (Cf. Plutarque, De Iside et Osiride; Aulu-Gelle, Noctes Atticae) des lieux communs, au même titre que les lieux communs obligatoires sur les voyages d'études qu'y auraient faits les philosophes. À l'époque de Platon, cependant, il est difficile d'admettre que la connaissance de l'Égypte ait été aussi largement répandue parmi les Grecs, et c'est pourquoi nous commencerons par chercher des éclaircissements sur cette question dans les Histoires d'Hérodote d'Halicarnasse; on sait en effet que, dans cet ouvrage, rédigé une génération environ avant la naissance de Platon, et qui fut un best-seller de l'époque, se trouve une large section (le livre II) intégralement consacrée à l'Égypte.

Hérodote, fils des Lumières ioniennes, s'intéresse à l'Égypte en tant que géographe, voyageur et anthropologue ${ }^{15}$. Il voit dans ce pays le don

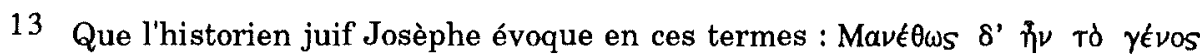

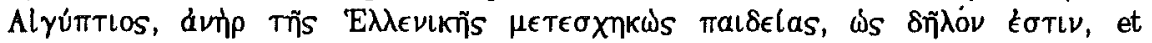
qu'il cite en entier in Contra Apionem I. 14, § 73-92; repr. in Manetho, ed. W ADDELL (Loeb Classical Library, Londres, 1940)

14 Le géographe Strabon savait déjà que le mot grec oasis venait de l'égyptien, mais c'est seulement au XXe siècle que son origine exacte a été connue : le mot égyptien wahet signifie "chaudron". "Désert» vient de l'égyptien $d s ̌ r t$, qui veut dire "rouge", un mot qui était opposé aux noirs $(k e \bar{e} m e(t))$ alluvions des rivages $\mathrm{du}$ Nil. Pour d'autres mots dans les langues modernes, pour lesquels on peut suspecter, avec plus ou moins de probabilité, une origine égyptienne (par exemple ébène, gomme, sac, lis, ibis, uraeus, phénix, basalte, albâtre, etc.) voir CERNÝ, ibid., p. 201-8. On hésite à assigner une origine grecque au mot "papyrus», puisqu'il n'est pas attesté avant Théophraste : voir à ce sujet Naphtali LewIS, Papyrus, in Classical Antiquity 4, n. 2.

15 Pour un jugement récent sur l'œuvre d'Hérodote, voir le livre de vulgarisation de R.P. Lister, The Travels of Herodotus, Londres, 1979, ainsi que les ouvrages de J.A.S. EvANS, Herodotus, Boston, 1982, qui consacre un chapitre à l'Égypte, et de J. HART, Herodotus and Greek History, Londres, 1982, sans oublier l'ouvrage collectif, Herodot. Eine Auswahl aus der neueren Forschung, 3e éd., Darmstadt, 1982 (Wege der Forschung, Bd. 26, hrsg. von W. MARG). On 
du Nil (une formule que reprendra Platon) et décrit avec force détails sa côte, son Delta, ses villes et son grand lac Moasis, ainsi que les conditions de vie en Haute-Égypte jusqu'à Éléphantine (Assouan). Il donne aussi une description précise de la fertilisation de cette terre par les inondations annuelles du fleuve. Et, s'il déclare avoir peu d'intérêt pour les questions religieuses (car à propos des dieux, pense-t-il, n'importe quel peuple en connaît tout autant ou tout aussi peu que

peut aussi trouver une interprétation originale de la perspective d'Hérodote dans C.W. FonNarA, Herodotus : An Interpretative Essay, Oxford, 1971. Les critiques n'ont évidemment pas manqué de s'interroger sur la valeur du témoignage d'Hérodote concernant l'Égypte, comme d'ailleurs sur celle de son œuvre historique en général. En ce qui concerne l'Égypte, les différents jugements des historiens ont été bien résumés et discutés par F. OERTEL, Herodots ägyptischer Logos und die Glaubwürdigkeit Herodots, Bonn, 1970. Oertel incline à défendre Hérodote contre l'accusation de syncrétisme culturel, en soulignant la réalité et les effets de la collaboration entre la Grèce et l'Égypte, qu'il appelle «eine gräkoägyptische Kultur-Koine» (p. 14): «So kommt es zu einem Synkretismus, der ägyptische und griechische Züge trägt, und dessen Niederschlag wir bei Herodot antreffen, und der dann modernen Skeptizismus wachgerufen hat, wenn nur griechisch mögliche Erzählungen von Göttern und Heroen, aber auch von Pharaonen wie Amasis, von Mysterien, Orakeln und dgl. in ägyptischen Gewändern oder ägyptischer Umgebung erscheinen. Griechen und Ägypter haben in der saïtisch-persischen Epoche eben vereint an der gemeinsamen Tradition, die ägyptische Königsgeschichte eingeschlossen, gearbeitet, wobei eine Menge Novellistisches und Folkloristisches sich angegliedert hat, der Grundstock aber doch ägyptisch bleibt». Une contribution intéressante à la discussion moderne des rapports entre la Grèce et l'Égypte se trouve dans l'article de D. MALLET, Les rapports des Grecs avec l'Egypte (521-331), in Mém. franç. d'arch. orient., 48 (1922). Toute la question du voyage d'Hérodote en Égypte a été réexaminée à nouveaux frais par A.B. LLOYD, Herodotus. Book II, Leyde, 1975, qui a aussi fourni le commentaire littéral le plus pénétrant paru jusqu'ici : Herodotus Book II .Commentary 1.98, Leyde, 1976. Bien que Lloyd admette (dans son ouvrage de 1975) que «there is good reason to suspect the handiwork of Herodotus in much of the chronology of Egypt and in the attempts to reconcile the contradictions which arose between that and traditional Greek chronology", son jugement est dans l'ensemble assez positif : "As for Egyptian chronology, Herodotus and his fellow researchers accumulated a large stock of information on the subject and found to their considerable embarrassment that its beginnings lay millenia before what they considered to be the beginnings of their own history in the sixteenth century B.C. It is the traumatic effect of this discovery which caused Herodotus to devote so much time to chronological problems in Book II. The details of his Egyptian chronology are surprisingly accurate» (p. 194). 
n'importe quel autre ${ }^{16}$ ), en réalité c'est loin d'être la cas, ainsi que nous le verrons plus loin.

La description de l'Égypte par Hérodote est trop longue, trop détaillée, trop colorée, et, dans certains cas, trop problématique, pour que je puisse en donner ici un résumé adéquat. Je me contenterai donc d'y relever les passages auxquels s'est référé Platon, dans le but d'en extraire les particularités qui peuvent éclairer le mythe égyptien de Platon. Ainsi Platon fait allusion, comme on l'a vu, à «la ville de Saïs, d'où venait le roi Amasis». Or Hérodote mentionne bien la ville et le nome de Saiis, et son livre sur l'Égypte s'achève en fait par une courte biographie du dernier pharaon ayant régné avant l'invasion (en 522 av. J.-C.) de Cambyse (Kambuziya), fils de Cyrus le Grand et roi de Perse entre 530 et $522^{17}$ : ce pharaon venait précisément de la ville de Siuph (dans le nome de Saïs) et portait le nom d'Amasis ${ }^{18}$. Il ne fait guère de doute que nous avons affaire ici à la source de Platon, ou au moins à l'une de ses sources. Ce lien étant établi, nous pouvons alors aborder les questions qui se posent à propos des idées d'Hérodote et de Platon sur l'Égypte et ses rapports avec la Grèce.

\section{La parenté supposée entre l'Égypte et la Grèce}

La déesse de Saïs, dit Platon, est appellée Neith en égyptien, "et en grec, à ce qu'ils [les Égyptiens] disent, Athéna"19. Les gens de Saïs, poursuit-il, sont très philathênaioi et revendiquent d'ailleurs une certaine parenté avec les Athéniens. Quel crédit peut-on accorder à ces remarques empruntées à la description d'Hérodote ? Voyons-en la première partie, à savoir l'identification opérée par Platon entre Neith

16 Hist. II, 3.

17 Hist. III, 7-15

18 Sa biographie commence en Hist. II, 163, et se poursuit jusqu'à la fin du livre II. Le début du livre III nous raconte la mort d'Amasis, juste avant l'invasion perse.

19 Platon affirme sans ambiguité (Timée, 21e) que les habitants de Saìs euxmêmes identifient Neith et Athéna et revendiquent leur parenté avec les Athéniens. Mais ici les traducteurs ne rendent pas toujours le grec avec toute la

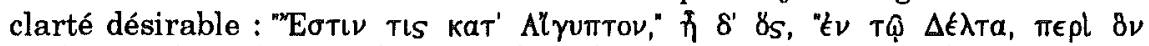

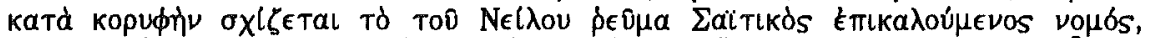

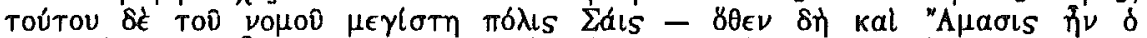

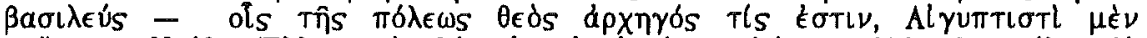

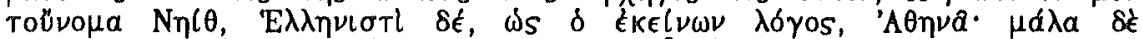

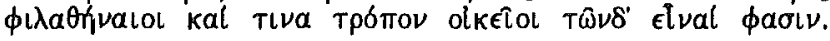


et Athéna. Hérodote, en dépit de ses affirmations sur son manque d'intérêt pour les questions religieuses, nous donne dans son œuvre un grand nombre d'informations sur les dieux et les déesses de l'Égypte. Bien plus, il identifie chaque divinité égyptienne avec une divinité équivalente du panthéon grec : ainsi Aphrodite correspond à Hathor, la déesse-vache de la fertilité; Osiris, «au dire des Égyptiens», est Dionysos (II, 42); Artémis est Bast; Déméter est Isis (II, 59); Athéna est Neith; Arès est Seth (ibid.); Horus est Apollon (II, 144); et enfin Ammon est le nom égyptien de Zeus (II, 42). Cependant, on observe çà et là des difficultés de détails qui troublent Hérodote, tant il prend au sérieux ce système d'identification. C'est ainsi qu'il se demande si les Égyptiens ont aussi comme héros l'autre Héraclès qui est vénéré par les Grecs, c'est-à-dire non pas le fils de Zeus/Ammon, mais bien le fils d'Amphitryon le héros. En effet, notre historien nous rappelle qu'Amphitryon et sa femme Alcmène étaient Égyptiens et qu'Héraclès fut emprunté aux Égyptiens par les Grecs, et non l'inverse. Et il poursuit en disant que Pan (= Min) fait partie de l'ogdoade égyptienne («les huit dieux d'origine») et qu'il est représenté avec la tête et les jambes d'un bouc, comme en Grèce. Nous commençons ainsi à entrevoir l'idée générale d'Hérodote. Lui-même d'ailleurs ne tarde pas à la formuler et à la justifier : les Égyptiens furent les premiers à nommer les dieux et les Grecs se mirent à leur école, en se servant de noms grecs au lieu de noms égyptiens. Qui plus est, les Grecs empruntèrent aux Égyptiens des cérémonies religieuses telles que le Festival de Dionysos (II, 48-50), qui était un sacrifice; Hérodote se demande même pourquoi la circoncision ne fut pas aussi adoptée. Il est donc évident qu'il considère les panthéons grec et égyptien comme strictement équivalents.

Dans le récit d'Hérodote, le thème de l'emprunt des Grecs en matière religieuse se confond avec celui de la jeunesse relative de la Grèce par rapport à l'Égypte (II, 54, 64,146). Notre historien n'admet qu'un seul emprunt en sens inverse, et encore ne s'agit-il que d'un emprunt mineur (II, 91). Mais il affirme aussi que les Égyptiens furent les premiers à avancer l'immortalité de l'âme et la réincarnation, toutes croyances qui furent adoptées plus tard par plusieurs auteurs grecs ${ }^{20}$.

Mais revenons à Platon. Son identification de la déesse Neith de Saiis avec Athéna n'est pas une remarque accidentelle. Car, quand nous la considérons à la lumière du récit d'Hérodote, elle nous permet

20 Il se trompe évidemment en ce qui concerne la réincarnation, car il ne s'agit pas là d'une croyance égyptienne. 
d'inférer que Platon acceptait probablement l'identification du panthéon grec avec le panthéon égyptien et qu'il considérait cette identification comme une particularité de la conscience religieuse des Égyptiens, ce qui, à ses yeux, expliquait sans doute la parenté que, selon lui, les gens du nome de Saïs revendiquaient avec ses compatriotes. Platon savait, probablement encore par Hérodote, que le roi Amasis venait de la province de Saïs. Son amitié envers les Grecs ne faisait aucun doute, car, dans sa biographie d'Amasis, Hérodote relate le mariage dynastique de ce roi avec une fille de la noblesse grecque de Cyrène (II, 180). Bien plus, Amasis "favorisa les Grecs», au point de leur léguer le port de Naucratis ("la reine de la mer") dans le Delta, pour qu'ils y établissent un comptoir commercial, et il leur accorda des terres pour l'érection d'autels et la construction de temples, dont le plus grand fut l'Hellenicon. La plupart des colons grecs étaient des Ioniens, des Doriens (comme Hérodote lui-même) ou des Éoliens, mais des relations existaient également avec les Grecs du continent, car Amasis offrit cent talents d'alun pour la construction du temple de Delphes. Athéna fit aussi largement l'objet de ses largesses : c'est ainsi qu'il fit parvenir à Cyrène une statue de cette déesse et un autoportrait; et au temple d'Athéna, à Lindos, deux autres statues. Le premier projet architectural d'Amasis fut, toujours selon Hérodote, le merveilleux portail du temple d'"Athéna" à Saïs. Tout ce que nous pouvons en conclure, c'est que l'intérêt que ce roi portait à Athéna présuppose évidemment qu'il acceptait effectivement l'identification de la déesse grecque avec Neith, déesse de sa province et de sa ville. De ce fait, dans sa description d'Amasis et de sa province natale comme "athénophiles» (philathênaioi), Platon est absolument fidèle à l'histoire. On peut dire qu'il s'est fondé sur la conscience que les Grecs avaient bel et bien de leur parenté avec l'Égypte, et que cette description n'est donc pas un produit de son imagination ${ }^{21}$.

À ce stade, il me faut encore dire un mot des autres emprunts que Platon peut avoir faits à Hérodote. Tout d'abord, le récit du Timée nous montre Solon voyageant dans le Delta et séjournant à Saïs, où il discute avec des prêtres de l'endroit sur des questions d'histoire. Or Hérodote rapporte que Solon emprunta à Amasis et intégra dans les lois d'Athènes l'admirable coutume selon laquelle «chaque homme devait une fois par an déclarer au nomarque (c'est-à-dire au gouverneur

21 Dans le Ménexène (245d), nous apprenons que les Grecs sont des descendants d'Égyptiens, mais il est difficile de savoir jusqu'à quel point le discours qui y est tenu est sérieux. 
provincial) la source de ses revenus, étant entendu que tout manquement à cette règle, de même que l'incapacité de prouver le caractère honnête de cette source, était puni de morts (II, 177). Il ne fait guère de doute que Platon connaissait bien le récit hérodotéen de la visite de Solon à Saïs.

En second lieu, le mythe ou le récit de Platon trace un parallèle entre les coutumes égyptiennes existantes et celles d'une Athènes archétypique : dans la structure hiérarchique de leur société, nous dit-on, les Égyptiens insistent aussi sur la séparation entre la caste sacerdotale et les autres classes, qui sont celles des artisans (y compris les bergers, les chasseurs et les fermiers) et des soldats (auxquels la loi interdit toute autre activité que la guerre). Or ceci s'accorde avec la description que donne Hérodote du système des sept classes qui était appliqué en Égypte (II, 166), et aussi avec son insistance sur le fait que les guerriers «ne se mêlent d'aucun commerce d'aucune sorte, mais reçoivent une éducation purement militaire, de père en fils», ce qui, ajoute notre historien, rapproche encore les Égyptiens des Grecs, qui, eux aussi, considèrent les guerriers comme la seule vraie noblesse ${ }^{22}$.

22 Isocrate souligne, pour en faire l'éloge, la stricte division en classes du peuple égyptien (voir Busiris, 15-17). Les caractéristiques remarquables de la politeia lacédémonienne sont, prétend-il, imitées de l'Égypte. Le Busiris offre de nombreux parallèles avec les remarques de Platon sur l'Égypte (son climat privilégié, 12; ses mérites artistiques, 17; sa pratique de la sagesse, 21; de la médecine, 33; des mathématiques et de l'astronomie, 23; la vraie piété et l'obéissance de ses citoyens, 28). Isocrate attribue l'origine de la philosophie grecque à une visite de Pythagore en Égypte et à son étude de la religion, du culte, des sacrifices et des cérémonies de ce pays. Certains voient une référence à la République dans la remarque d'Isocrate disant que «dans l'organisation qui fait durer la royauté et toutes leurs institutions politiques, ils ont eu un tel succès que les philosophes qui s'occupent de ces questions et y ont acquis la plus grande réputation, privilégient la constitution de l'Égypte...» (17) (trad. Mathieu-Brémond légèrement modifiée). Toutefois, si le Busiris fut composé vers $390-385$, cette allusion ne saurait évidemment se rapporter à la République. Notons aussi que dans sa Politique (VII, 1329 c 40 sq.), Aristote évoque la spécialisation des classes sociales en Égypte en ces termes : «Voilà donc où prit naissance l'organisation des repas en commun; la séparation du corps civique en classes, elle, vient d'Égypte, car la royauté de Sésostris est d'une époque de beaucoup antérieure à celle de Minos» (trad. Aubonnet). En ce qui concerne Naucratis, des fouilles archéologiques ont montré la présence de Grecs à cet endroit à partir des dernières années du VIIe siècle av. J.-C., c'est-àdire bien avant le règne d'Amasis. Voir à ce sujet l'article Naucratis dans The Oxford Classical Dictionary, p. 722-723. Pour les témoignages archéologiques 
Inutile de dire que les castes ne faisaient pas partie du système social primitif de l'ancienne Égypte, mais qu'elles relèvent d'une forme de société plus tardive et sclérosée ${ }^{23}$, si bien que l'idée de Platon, selon laquelle le système en question aurait été un élément originel de la structure sociale de l'Égypte, est manifestement fausse. Mais l'auteur de la République pouvait difficilement en avoir une meilleure connaissance, vu l'état de la science historique à son époque. D'ailleurs, Hérodote non plus n'en avait aucune idée.

\subsection{Les idées de Platon sur l'antiquité de l'Égypte}

Venons-en maintenant au thème, commun à Hérodote et Platon, de l'antiquité des Égyptiens par rapport aux Grecs. Comme nous l'avons déjà vu, chez Hérodote, ce thème apparaît sous la forme de l'idée que les Grecs auraient emprunté leur panthéon aux Égyptiens et que ceux-ci auraient été les premiers à distinguer les différentes divinités de ce panthéon, sans qu'il y ait eu d'influence dans l'autre sens. C'est qu'Hérodote, comme tous les anciens visiteurs de l'Égypte, avait été profondément impressionné par l'antiquité des monuments et par la longueur de la liste des pharaons. Il fut ainsi une victime consentante de l'orgueil des prêtres égyptiens à l'égard de leur propre culture, et il se laissa convaincre par eux que toutes les connaissances existant dans les autres pays tiraient leur origine de l'Égypte. Aussi défendit-il l'idée, sans faire preuve de beaucoup d'esprit critique, que le développement plus tardif de la civilisation en Grèce avait eu lieu sous l'influence de l'Égypte, y compris dans les arts du calendrier, de la divination et de l'astrologie.

Quant à Platon, c'est sur l'opposition entre le grand âge de la civilisation de l'Égypte et la jeunesse de la culture grecque qu'il construit tout son mythe égyptien : le peuple d'Égypte, dit-il en substance, a une

concernant le site de Naucratis, voir J. BoARDMAN, The Greeks Overseas, nouv. éd. augm., Londres, 1983, p. 118 sq.

23 Sur les caractéristiques générales de l'Égypte à l'époque saitique, y compris son archaïsme typique dans les domaines de l'art et de la religion, sa nostalgie de la royauté primitive, l'impact des influences étrangères et la séparation des castes, voir A. GARDINER, Egypt of the Pharaohs : An Introduction, Oxford, 1961, p. 355-356. Voir aussi la contribution de A.B. LLOYD, in B.G. TRIGGER, B.J. Kemp, D. O'ConNor et A.B. Lloyd, Ancient Egypt : A Social History, Cambridge, 1983, qui comporte une abondante bibliographie. Le livre II d'Hérodote est bien sûr essentiellement une description de l'Égypte de cette époque. 
histoire incomparablement plus longue que le peuple grec. C'est que le temps progresse selon des cycles, dont le commencement et la fin sont marqués par une catastrophe naturelle ou par l'achèvement de la Grande Année. Le phénomène de la précession des équinoxes, auquel se réfère Timée $22 \mathrm{c}$, est interprété comme le signe de la fin d'un grand cycle dans l'histoire de l'humanité, c'est-à-dire comme une véritable «révolution», après laquelle doit commencer une ère nouvelle. De ce fait, les réalisations des hommes au cours du temps sont éphémères et toujours menacées de destruction. Aussi chaque âge doit-il tout reconstruire, en commençant par produire les biens de première nécessité, avant de développer une culture et une vie politique. Seule l'Égypte a été épargnée, parce qu'elle est «le don du Nil» et qu'elle reçoit l'eau, non point du ciel, mais d'en bas (du fleuve même), ce qui la met relativement à l'abri des catastrophes ${ }^{24}$. C'est cela qui explique que sa mémoire historique remonte à quelque huit mille ans en chiffres symboliques, c'est-à-dire jusqu'à l'époque de la dynastie thinite ${ }^{25}$. Dès lors, il n'est guère étonnant qu'avec l'ancienneté et la continuité extraordinaires de leur culture, les prêtres égyptiens aient laissé une forte impression sur les Grecs, encore tout jeunes dans leur propre civilisation : comme le dit Hérodote, «avant le règne de Psammétique, les Égyptiens avaient coutume de penser qu'ils étaient la plus ancienne race du monde..., mais, depuis cette époque, ils croient que les Phrygiens les surpassent en ancienneté et qu'eux-mêmes viennent en second» (II, 1).

La mémoire historique des Grecs ne pouvait évidemment rivaliser avec une ancienneté de cet ordre, et du reste elle ne le fit point, elle dont le plus lointain passé, au demeurant de nature mythique, ne remontait pas plus haut que la civilisation minoenne, les légendes des guerriers et marins mycéniens, et les œuvres des grands poètes Homère et Hésiode, lesquels, "environ quatre cents ans avant mon époque", dit Hérodote, avaient combiné et codifié les différentes traditions antérieures. Ainsi, déjà bien avant l'époque de Platon, l'Égypte était profondément entrée dans la conscience grecque comme critère absolu de longévité culturelle et comme mère des sciences, des arts, de la divination et de la magie. Tout ce que les Grecs pouvaient faire était de revendiquer une filiation avec l'Égypte, car rivaliser avec elle eût été une vaine entreprise. À

24 Cf. HÉrodote, Hist. II, 13.

25 En fait, on sait que l'unification des deux royaumes égyptiens sous la souveraineté du roi Ménès, fondateur du pays ainsi unifié, remonte à environ trois mille ans avant J.-C. 
l'époque classique, ils regardaient l'Égypte à peu près comme nous regardons nous-mêmes le passé de notre civilisation, et comme nous parlons de notre héritage gréco-romain dans le domaine de la culture ou de notre héritage judaïque dans le domaine de la foi. Cette manière de voir le passé était elle-même un acquis, car elle permettait d'évaluer et de questionner les classiques à nouveaux frais. Il n'y a guère de philosophes importants, de Thalès à Platon lui-même, en passant par Pythagore et Démocrite, auxquels on n'ait attribué un voyage d'étude en Égypte. Aussi sujettes à caution que soient ces traditions doxographiques, elles témoignent d'une mentalité largement répandue, qui n'a pas attendu l'époque alexandrine pour se manifester, car elle existait déjà chez Hérodote, Isocrate et Platon, qui y ajoutèrent du reste leurs contributions personnelles. Aristote lui-même devait poursuivre dans la même voie. Paul Friedländer remarque à juste titre que "pour Platon, l'Égypte était un exemple étonnant de vie culturelle et politique immuable, contrastant avec le changement incessant propre au style de vie des Grecs; dans le monde systématiquement hiérarchisé de Platon, elle devint une entité politique située quelque part entre Athènes et l'État idéal» 26 .

Nous ferions cependant une erreur grossière si nous supposions qu'Hérodote fut la seule ou même la principale source de Platon concernant l'Égypte. En effet, plus nous examinons les écrits mêmes de Platon, plus nous voyons qu'il possédait une connaissance solide de l'Égypte. Sans doute cette connaissance ne se manifeste-t-elle le plus souvent qu'accidentellement, quand ce n'est pas sous la forme d'un badinage; mais, en tout état de cause, elle nous montre l'intérêt très vif que le philosophe athénien portait à cette vieille culture. À son époque, les Grecs d'Ionie et du continent avaient depuis longtemps fondé des colonies dans toutes les directions, sous la pression conjuguée de leur besoin d'espace et de leurs ambitions commerciales. Plus précisément, à partir du VIIIe siècle av. J.-C., ils avaient créé des établissements coloniaux dans le Pont-Euxin, autour de l'Hellespont et au nord de la mer Égée ${ }^{27}$. Depuis la fin du VIIe siècle, ils étaient solidement implantés en Sicile et en Italie méridionale (dans le Golfe de Tarente). Au départ de la colonie de Pithecuses, fondée au VIIIe siècle dans une île de la baie de Naples, il s'étaient installés sur la terre ferme et avaient

26 P. FRIEDLÄNDER, Plato, vol. I, p. 201.

27 Cf. J.B. Bury et R. MeIgGs, A History of Greece to the Death of Alexander the Great, 4e éd., Londres, 1975, ch. 2. 
fondé Néapolis et Cymé (où, pense-t-on, ils reçurent pour la première fois le nom collectif d'«Hellènes», car jusqu'alors ils étaient dénommés suivant les noms de chaque tribu). Les Phocéens s'étaient aventurés plus loin encore sur la mer, jusqu'à fonder Massilia vers 600, et d'autres établissements sur les côtes de Corse et d'Espagne. L'Égypte, en revanche, leur était restée fermée jusqu'à la fin de la domination assyrienne, qui avait suivi la conquête du pays par Assarhaddon. Mais, après une génération d'occupants assyriens, un général d'origine lydienne, Psammétique de Saïs, avait enrôlé en Ionie et en Carie des mercenaires pour l'aider à déloger ces occupants. C'est ainsi que Saïs était devenue la capitale d'une Égypte enfin réunifiée. De leur côté, les mercenaires grecs avaient été maintenus sur place et renforcés, tandis que, dans leur sillage, des commerçants de même nationalité avaient été autorisés à s'installer dans le pays, où ils avaient obtenu une concession permanente. Celle-ci, devenue la cité grecque de Naucratis, s'était développée à l'embouchure du canal occidental (ou «canopique») du Delta.

Ainsi, à l'époque de Platon, l'Égypte, ou à tout le moins le Delta, avait cessé depuis longtemps d'être une contrée étrangère pour les Grecs $^{28}$. De fait, Platon lui-même fait mention de Naucratis (Phèdre, 247d). Et il possède aussi des connaissances sur la haute Égypte, car il écrit que la cité appelée Thèbes en grec (Karnak) est la patrie du dieu Ammon et une ancienne capitale de l'empire. Il connaît bien la coutume égyptienne d'élever dans des étangs les poissons tirés du Nil

28 Pour un aperçu équilibré sur les relations entre les peuples d'Égypte et de Grèce, ainsi que sur les jugements qu'ils portèrent les uns sur les autres aux différentes périodes de leur histoire, depuis les temps homériques jusqu'à l'ère chrétienne, voir la leçon inaugurale du Prof. J.W.B. BARNS à Oxford : Egyptians and Greeks, Bruxelles, 1978 (Papyrologica Bruxellensia, 14). Sur les relations antérieures entre les peuples de la mer Égée et l'Égypte, voir Die Beziehungen Ägyptens und Vorderasiens zur Ägäis bis ins 7. Jhdt. v. Christus, Darmstadt, 1979 (Erträge der Forschung, Bd. 120). Récemment, Martin BERNAL a lancé un défi à la thèse, aujourd'hui généralement admise, selon laquelle les Grecs étaient essentiellement des Européens ou des Ariens, et il a essayé de remettre en honneur la vieille thèse selon laquelle la culture grecque aurait été le résultat d'une colonisation de la Grèce par les Égyptiens et les Phéniciens, qui, aux environs de 1500 av. J.-C., auraient commencé à se mêler avec les indigènes. Son livre, fort controversé, porte le titre provocateur de Black Athena. The Afroasiatic Roots of Classical Civilization. Le volume I, The Fabrication of Ancient Greece 1785-1985, a paru à Londres en 1987. Le volume II, qui contient l'essentiel de l'argumentation de l'auteur, a paru en 1991 : The Archaeological and Documentary Evidence. 
(Politique, 264c). Incidemment, il cite le prix du voyage du Pirée en Égypte : deux drachmes (Gorgias, 511d). Il connaît aussi, bien sûr, la pratique de la momification, puisque dans le Phédon (80c), Socrate parle des efforts des Égyptiens pour arrêter la corruption du corps au moyen de l'embaumement. Dans le Ménexène, il rappelle la conquête de l'Égypte par Cyrus et celle de la Libye par le fils de celui-ci (239e), de même que l'expédition lancée par les Grecs pour aider l'Égypte à l'époque de la guerre avec la Perse (241e). Il a également des idées sur les «enfants du Nil», qui ne sont pas toujours flatteuses, mais qui reflètent sans doute les préjugés courants des Grecs de l'époque : c'est ainsi qu'il voit dans l'amour de l'argent un trait typique des «habitants de l'Égypte», alors qu'il fait de l'amour du savoir la principale caractéristique des Grecs (Rép. IV, 435d-436a). Par ailleurs, Platon blâme la rouerie des Phéniciens et des Égyptiens, dont la cause serait, selon lui, le caractère peu libéral de leurs occupations et leur cupidité mercantile (Lois $\mathrm{V}$, 747c). De plus, ils manquent tout à fait aux lois de l'hospitalité (Lois XII, 953e). Cependant, dans d'autres domaines, notre philosophe tient l'Égypte pour un modèle : un cas typique est l'intelligence dont les Égyptiens font preuve en enseignant l'arithmétique à leurs enfants, car pour ce faire ils utilisent des jeux (Lois VII, 819b). Bien entendu, les autres motifs de louange sont plus substantiels et en rapport plus étroit avec notre discussion, mais j'y reviendrai plus loin.

Une dernière remarque historique : Pindare mentionne déjà la colonie Théréenne de Cyrène, près de la côte libyenne, le seul établissement grec d'Afrique du Nord qui soit parvenu à quelque célébrité et à la prospérité. Cyrène, en effet, avait acquis un renom particulier du fait qu'elle contrôlait l'accès de l'oasis de Siwa, laquelle était située dans le désert intérieur et abritait un oracle d'Ammon, qui jouissait auprès des Grecs d'une réputation égale à celle des oracles de Delphes et de Dodone ${ }^{29}$. On trouve chez Platon plusieurs références à cet oracle. Peutêtre sont-elles à l'origine de la croyance, partagée par Diogène Laërce et Apulée, selon laquelle Platon aurait visité Cyrène. Tout au début du Politique, Théodore jure "par notre dieu Ammon». C'est que Théodore est de Cyrène, où cette exclamation était l'équivalent de l'expression grecque courante "par Zeus». Dans les Lois V, 738b-c, Platon mentionne les trois grands oracles de Delphes, de Dodone et d'Ammon; et nous

29 Hérodote raconte deux histoires pour expliquer comment les oracles de Dodone et de Siwa dérivent de la Thèbes d'Égypte (II, 55). Pour une étude générale et détaillée des trois grands oracles, voir H.W. PARKE, The Oracles of Zeus , Oxford, 1963. 
savons que le dernier nommé, celui de Siwa, était souvent consulté par les Grecs continentaux de son temps, comme par exemple Lysandre, mort en 395. À l'époque même de Platon, une des nefs utilisées pour les cérémonies religieuses était appelée "Ammonis", d'après Ammon, et un sacrifice public était offert à ce dieu par les magistrats d'Athènes ${ }^{30}$. Hérodote s'intéresse évidemment à Siwa et à l'oracle d'Ammon; mais, c'est, je présume, parce que ses lecteurs souhaitaient toujours en entendre parler par quelqu'un qui avait visité la côte africaine, et nous ne devons pas supposer qu'il fût la seule source d'information de Platon, tant s'en faut. Car chaque Athénien le connaissait, puisque l'Égypte était largement présente à l'esprit des Athéniens, depuis qu'une expédition navale de quelque deux cents galères avait été envoyée pour soutenir la révolte des Égyptiens contre la Perse en 459 av. J.-C., et qu'elle avait réussi à prendre Memphis. Comme nous l'avons vu plus haut, Platon mentionne d'ailleurs cette expédition dans le Ménexène (241e), peu après avoir évoqué la conquête de l'Égypte par les Perses $(239 \mathrm{e})^{31}$.

Ajoutons qu'en plus d'Ammon, qui était la grande divinité de Thèbes et l'une des plus importantes figures du panthéon égyptien (au moins à partir de l'époque des rois de la XVIIIe dynastie : vers 1567-vers 1320 av. J.-C.), Platon connaissait bien Thot, le dieu à l'ibis qui était vénéré comme patron de la grammaire et de la phonétique (Philèbe 18b), ainsi que du calcul, de l'astronomie, des jeux et de l'écriture ${ }^{32}$. Que

30 Voir les articles Ammon dans The Oxford Classical Dictionary, p. 51-53, et dans le Lexikon der alten Welt, Zurich/Stuttgart, 1965, col. 138-139.

31 Qui est ici faussement attribuée à Cyrus. Friedländer pense que le manque délibéré de soin dans les détails est un trait caractéristique de certains des discours d'éloge d'Athènes dans le Ménexène.

32 Dans le Phèdre (274c-275b), Socrate raconte à Phèdre l'histoire du dieu Theuth (Thot), qui vécut près de Naucratis et dont l'oiseau sacré est l'ibis. Selon le récit de Socrate, Theuth inventa non seulement l'arithmétique, la géométrie, l'astronomie, le trictrac et les dés, mais aussi et surtout l'écriture. Theuth vint voir Thamous (qui est, à mon avis, une forme hellénisée de Touthmôsis), qui «en ces temps-là régnait sur l'Égypte entière, dans cette grande ville du haut pays que les Grecs appellent Thèbes d'Égypte, et dont ils nomment le dieu Ammon". Le roi fit diverses remarques, positives ou négatives, sur la valeur de chacun de ces arts, mais, arrivé à l'écriture, il nia fermement qu'elle fút une potion magique pour améliorer la mémoire des Égyptiens, et affirma qu'elle découragerait plutôt l'exercice de la mémoire, au profit d'une confiance aveugle dans les souvenirs écrits, et qu'elle donnerait ainsi à ses utilisateurs l'illusion de la science, plutôt qu'une science réelle. Cette histoire ne nous 
notre philosophe ait ou non personnellement visité l'Égypte, ses allusions à ce pays témoignent d'une vaste connaissance de celui-ci, mais pour moi une telle connaissance était le propre de tout Athénien cultivé.

Si cet article n'avait été écrit que pour des historiens, j'aurais pu m'arrêter ici, ou poursuivre l'enquête sur les origines des mathématiques grecques, ou encore étudier l'influence de l'Égypte sur le sens grec de la forme. Mais Platon est un philosophe de génie et un poète, qui a choisi ses matériaux avec soin et qui les a organisés en ayant à l'esprit une idée bien précise. C'est cette idée que nous devons nous efforcer de découvrir en analysant le produit de son art. Aussi dirai-je maintenant quelques mots, à titre purement indicatif, du passage du Timée (24b-c) qui porte sur les lois égyptiennes et la sagesse qu'elles recelaient aux yeux de Platon.

\subsection{Les lois égyptiennes}

Quand Platon parle de lois, nous ne devons pas penser à une législation au sens moderne du terme, mais plutôt à tout l'idéal historique d'une communauté, qui se réalise dans un consensus social et s'exprime dans une constitution, dont le but est de servir et de maintenir le bon ordre de cette communauté. Ce que nous voyons en premier lieu dans le Timée, c'est que Platon loue la constitution égyptienne pour

intéresse ici que par la connaissance dont elle témoigne des réalités et des croyances égyptiennes. Ainsi voyons-nous qu'elle joue sur les idées populaires que les Grecs avaient sur l'Égypte, puisqu'ils voyaient justement en celle-ci un pays d'une vénérable antiquité, où les symboles écrits revêtaient un caractère ouvertement sacré et étaient d'un usage très ancien, et où aussi fleurissaient la magie et les potions magiques (275a). Certes, Phèdre taquine Socrate avec ces mots : «Quelle aisance tu as (...) à composer des histoires égyptiennes, ou de n'importe quel pays, selon ton bon plaisir !». Mais il s'attire aussitôt la remarque de Socrate que pour lui, Phèdre, «l'important est sans doute de savoir qui parle, et quel est son pays d'origine», alors que l'essentiel est bien plutôt «de savoir s'il dit, ou non, les choses comme elles sont" (trad. Vicaire). Le style enjoué du passage dissimule, à mon sens, sa nature ironique : les Égyptiens ont, en effet, utilisé l'écriture depuis la plus haute antiquité, mais cela n'en a point fait ni ne pouvait en faire des philosophes! De ce point de vue, l'histoire conduit naturellement à l'examen dialectique de la différence qui existe entre le discours *qui s'écrit avec la science dans l'âme de l'homme... le discours vivant et animés (276a), d'une part, et le discours inerte et sans réponse qui s'écrit sur le papier, d'autre part. La supériorité du discours vivant sur le discours écrit rejoint et prolonge le premier thème du dialogue, de la supériorité de la dialectique sur la rhétorique. 
l'importance immémoriale qu'elle a toujours accordée à la sagesse (phronèsis), en tant que celle-ci se trouve dans l'ordre du monde et qu'elle a permis, en partant des réalités divines, d'inventer tous les arts utiles à la vie, y compris la divination et la médecine ${ }^{33}$. Aussi y a-t-il dans les lois égyptiennes quelque chose comme un élément sacré, comme une tentative de déduire l'action humaine d'un ordre du monde divin et paradigmatique, et d'établir par là un lien étroit entre la société et la nature, avec pour corollaires la permanence et la résistance à la corrosion du temps. Sur ce point, la réflexion de Platon va beaucoup plus loin que les observations d'Hérodote, qui se situent à un niveau nettement inférieur d'expérience et d'analyse. Car avec l'auteur du Timée, nous entrons dans le royaume de la pensée créative et nous sommes loin des problèmes de sources et d'influence. C'est dire qu'il faut s'efforcer d'approcher ce grand philosophe à son propre niveau. Heureusement, nous disposons pour ce faire de l'aide inestimable d'un grand interprète de la pensée philosophique de Platon qui a longuement réfléchi au sens de son mythe égyptien, je veux parler d'Éric Voegelin ${ }^{34}$.

Dans quelle mesure Platon a-t-il bien ou mal compris la justification sacrale, par les Égyptiens, de leur culture et de leurs activités ? Son allusion au fait que les arts dériveraient de l'ordre du monde est-elle correcte, ou, au contraire, abusive ? Les réponses à de telles questions sont nettement du ressort de l'égyptologie et doivent être tirées de sources qui sont multiples et variées, mais peu systématiques et de nature souvent mythologique ou pré-philosophique. Les remarques qui suivent ne prétendent nullement faire autorité du point de vue de la science historique. Toutefois, dans le contexte de notre discussion, nous essayerons tout de même d'esquisser à grands traits les croyances égyptiennes concernant l'ordonnancement divin des réalités fondamentales, la

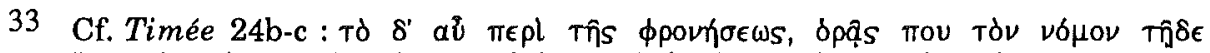

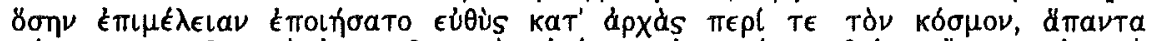

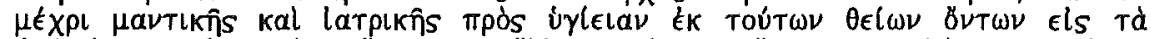

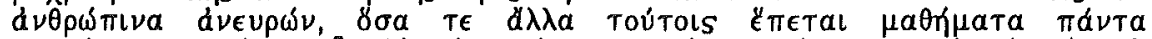

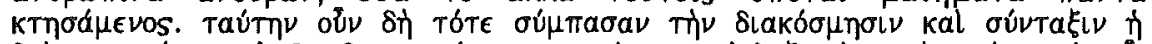

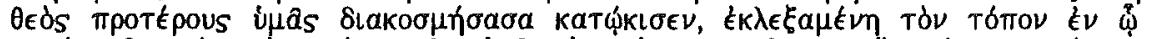

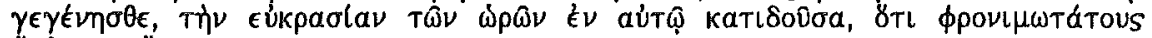
¿uspas olool.

34 Cf. E. Voegelin, Plato's Egyptian Myth, in The Journal of Politics, 9 (1947), p. 307-24. Voir aussi, du même auteur, Plato and Aristotle (Order and History, vol. III), Bâton Rouge, 1957, p. 170-80. 
nature de l'ordre social de l'Égypte même, ainsi que la solidarité qui existait, aux yeux des Égyptiens, entre l'ordre divin et l'ordre social ${ }^{35}$.

\section{L'ordre égyptien}

En Égypte ancienne, les formes de la vie religieuse étaient aussi nombreuses que variées : mentionnons, entre autres, les prières pour les vivants, les textes magiques pour la survie des morts, les hymnes du culte du temple, les généalogies des dieux, ainsi que les textes se rapportant au couronnement et aux funérailles des pharaons. Toutes ces manifestations religieuses s'adressaient à une multitude de dieux et de déesses (certains parmi eux revêtant, comme on sait, la forme d'animaux) et elles visaient à développer une relation harmonieuse et intime entre les dieux supérieurs et le soleil. C'est pourquoi on s'adressait au dieu-créateur Ammon, en tant qu'Ammon-Râ, et Râ revêtait lui-même d'autres formes, telles que Horakté, le dieu à tête de faucon du soleil levant. Particulièrement intéressants pour notre propos sont les parallèles élaborés qui étaient tracés entre l'ordre des cieux et l'ordre de la société égyptienne. L'expression de l'ordre politique au moyen de l'analogie avec l'ordre cosmique était un trait commun à toutes les "religions» du Proche-Orient ancien ${ }^{36}$. Elle avait pris une forme caractéristique très tôt dans l'histoire égyptienne, peut-être dès l'époque de l'union de la haute et de la basse Égypte, sous le premier roi, Ménès, au seuil du troisième millénaire av. J.-C.

Pour les Égyptiens, un ordre unique enveloppait toutes choses. Sa force était divine et rayonnait des dieux vers le monde. C'est elle qui avait arraché la terre au chaos primitif des eaux et avait formé une hauteur sacrée (le symbole de la terre d'Égypte, qui s'exprimait dans la hauteur des pyramides). C'est elle aussi qui fondait l'autorité souveraine du pharaon. Les symboles sous lesquels s'exprimaient ces croyances, sont si denses et si complexes que notre pensée, pourtant beaucoup plus différenciée, ne parvient pas à s'élever à leur niveau. En effet, habitués comme nous le sommes à distinguer clairement entre Dieu, le monde, la société et l'homme - les quatre grands domaines selon lesquels l'être a été différencié par des siècles de Révélation chrétienne et de philosophie -, nous devons presque nous avouer vaincus

35 Pour ce qui suit, je m'inspire directement de E. Voegelin, Egypt, in Israel and Revelation (Order and History, vol. I), p. 52-110, qui est une synthèse intelligente des diverses constructions des plus éminents égyptologues. 
devant la densité de symboles tels que la couronne, la pyramide, la théologie memphite ou la barque-soleil, car ce sont là autant de signifiants qui sont à la fois théologiques, cosmologiques et politiques. Pris tous ensemble et dans toutes les surdéterminations de leur sens, ces symboles formaient une sorte d'ordre qui constituait pour les Égyptiens un champ sémantique à l'intérieur duquel leur vie individuelle s'inscrivait tout naturellement. Cet ordre était à la fois celui de la justice (placée sous le pharaon) et celui de la vie (placée sous l'œil d'Horus, ce soleil idéal, qui, après avoir vengé son père Osiris, avait reçu l'Égypte en héritage). Les Égyptiens croyaient aussi que, par l'intermédiaire de la figure divine du pharaon, l'ordre du cosmos rayonnait dans la société à la manière dont le soleil répand ses rayons sur la terre. Cette croyance était si forte et si stable qu'elle survécut à de longues périodes d'invasions et de troubles intérieurs, car, malgré l'expérience amère qu'ils firent alors de la détresse, du déshonneur et du désordre, les Égyptiens ne purent renoncer à l'idée qu'une existence prospère et heureuse devait nécessairement avoir pour cadre l'ordre vrai des choses qu'était pour eux l'ordre pharaonique, si bien que le retour de celui-ci fut ardemment désiré durant ces périodes d'insécurité.

Dans un des textes retrouvés à l'intérieur des pyramides de l'Ancien Empire, le statut véritablement divin du pharaon est énoncé dans toute sa pureté, quand, après sa mort, les dieux souhaitent la bienvenue au roi mort ${ }^{37}$ :

Voici mon fils, mon premier-né...

Voici mon bien-aimé en qui je me complais.

Voici mon bien-aimé, mon fils;

À lui, j'ai donné les horizons, pour qu'il les domine de

toute sa puissance, comme Horakté.

Il est vivant, roi de la Haute et de la Basse-Égypte, bien-aimé de Râ, vivant à jamais.

La loi du fils de Dieu canalise les forces divines et cosmiques vers l'intérieur de la société, de sorte que son couronnement est la répétition symbolique de l'ordonnancement originaire du monde par Dieu. Au moment de son couronnement, le roi est appelé à assumer le rôle du

37 J'ai donné ici une traduction française littérale des vers extraits de S.A.B. Mercer, The Pyramid Texts in Translation and Commentary, vol. I, New York, 1952. J'ai regroupé trois vers à partir d'un même contexte : $1 \mathrm{a}-\mathrm{b}, 4 \mathrm{a}-\mathrm{b} ; 6$. La traduction anglaise rappelle beaucoup Mt. 3,17, peut-être trop... 
dieu-créateur, en se maintenant sur l'éminence de terre qui émergea la première des eaux informes et qui se confond avec le royaume des deux pays $^{38}$ :

Tiens-toi debout sur elle, sur cette terre, qui sort 'd'Aton...

Tiens-toi au-dessus d'elle; Tiens-toi bien haut au-dessus

d'elle, que ton père puisse te voir; que Râ puisse te voir. Il est venu à toi, son père;

il est venu à toi, Râ.

La montée sur le trône mime la montée paradigmatique du dieuartisan au sommet de l'ordre, symbolisé par les pyramides et par le tracé des temples d'Égypte, lesquels s'élevaient par paliers vers l'autel situé au sommet. Après cette vie terrestre (car le pharaon mourra, comme toute l'espèce à laquelle il appartient), son $k a$ s'élèvera jusqu'à étreindre son père divin, dont la seigneurie éternelle se manifestait sur terre par sa médiation et continuera à se manifester par la médiation de ses successeurs. L'émergence contemporaine de l'ordre du monde et de l'ordre pharaonique est à la base de la salutation suivante d'un pharaon du Bas Empire, Thoutmosis III (vers 1500-1450) ${ }^{39}$ :

Qui est le roi de la Haute et de la Basse-Égypte ?

II est un dieu grâce auquel on vit, le père et la mère de tous les hommes, par lui-même unique, sans égal.

Dans la personne du pharaon se manifeste à la fois le créateur, le père divin et la déesse $M a \hat{a} t$, qui, pareille à la lumière du soleil, représente l'ordre des dieux, lequel dispense ses rayons dans chaque partie du pays, ainsi que la justice et la vérité religieuse (on peut en effet trouver tous ces sens dans le riche concept de $M a \hat{a} t^{40}$ ). Dans l'établissement de l'ordre juste, le pharaon est aidé par ses vizirs et ses

38 Ibid., vers 199 a - 200 a.

39 Cette inscription se trouve dans le tombeau de Rekhmire, le vizir de Touthmôsis III, et elle est citée par H. FrankForT, Ancient Egyptian Religion, New York, 1948, p. 43.

40 Fille de Râ et épouse de Thot, en même temps que déesse de la Sagesse, de la Vérité, de la Justice, de la Loi et de l'ordre, Maât était représentée sous la forme d'une figurine d'offrande dont la tête était surmontée d'une plume d'autruche qui devait servir, dans le plateau de la balance d'Osiris, à équilibrer le cœur humain placé dans l'autre plateau. Elle représentait l'ordonnance de l'univers, la morale, la justice et la vérité, qui concourent à l'harmonie des éléments du monde. 
gouverneurs, qui transmettent ses ordres à travers tout le royaume; mais eux-mêmes ne se considèrent dans leurs différentes fonctions que comme des porteurs de la substance une et immuable de la royale et divine Maât.

La densité de ces symboles résiste certes à toute transcription dans le langage clair et rationnel de l'expérience humaine des civilisations modernes. Mais elle possédait une clarté propre à elle, la clarté, pourrait-on dire, du soleil et du roi-soleil, ceux-ci étant tous deux la manifestation de l'unique puissance ordonnatrice, qui, partant des dieux, circulait dans toute la nature et toute la société, avant de revenir à sa source. Cette puissance assurait l'intégration, aussi bien de l'homme dans la société, que de la société elle-même dans l'ordre cosmique, et, pour cette raison, elle se révéla extrêmement résistante à toute réorientation de l'existence que la dégénérescence de l'ordre empirique avait pu produire lors de périodes de troubles (qu'on appelle couramment les "époques intermédiaires», et qui suivirent l'une l'écroulement de l'Ancien Empire [vers 2700-2200 av. J.-C.], et l'autre celui du Moyen [vers 2050-1800]).

\section{L'Égypte et l'Idée platonicienne}

Maintenant que nous avons bien en tête ces idées générales sur les rapports entre la société et le cosmos, ou entre la justice et l'ordre divin du monde en Égypte ancienne, nous sommes mieux armés pour aborder le récit égyptien de Platon et pour l'utiliser comme une clef pour comprendre, aussi bien la vision que notre philosophe avait de ce pays, que sa pensée la plus élaborée concernant la réalisation de son idéal (c'est-à-dire, pour lui, de la vraie réalité) dans l'ordre temporel. En ce qui concerne, tout d'abord, le contexte philosophique et littéraire du récit égyptien, je peux dire d'emblée que j'approuve et adopte l'opinion de Voegelin sur la continuité entre la République et le Timée. Dans la République, l'idée de la polis apparaît comme un paradigme, mais son statut comme ordre d'une polis réelle n'est jamais entièrement clarifié :

En utilisant un terme moderne, on pourrait peut-être dire de la description de la polis bien ordonnée qu'elle est une "projection" de l'âme bien ordonnée. Les difficultés concernant le statut de l'idée sont ce qui a conduit Platon de la République au Timée. Du point de vue théorique, la République s'achève sur une grande question. Et c'est cette question qui reçoit une réponse dans le Timée (...), par le biais d'un mythe qui fait passer la polis bien ordonnée dont il 
est question dans la République, du niveau d'un simple récit au niveau d'un ordre inscrit dans une réalité historique. 41

La solution doit donc se trouver dans un cadre historique. Mais lequel ? Platon n'avait pas à sa disposition l'idée judéo-chrétienne d'une histoire orientée vers une fin transcendant le monde, ni la notion hégélienne de dialectique historique. Il devait donc constituer son cadre historique en se servant de la conception du temps la plus haute et la plus noble dont il disposait, c'est-à-dire la conception selon laquelle le temps se développe en cycles qui imitent le mouvement cyclique du cosmos. Dès lors, l'histoire devait chez lui être interprétée par analogie avec la nature et le rythme cosmique, c'est-à-dire par référence à ce qu'on pourrait appeler le mythe cosmologique.

Le mythe égyptien de Platon apparaît ainsi, dans son sens profond, comme un récit liant l'idée (ou la réalité, pour le dire simplement) au monde du temps, de la naissance et de la mort. Remarquons au passage la présence dans le mythe du thème de l'anamnèse, auquel Voegelin a consacré toutes les ressources magistrales de son savoir-faire d'exégète : Critias, le narrateur, a oublié le récit qu'il a entendu dans sa jeunesse et il doit le retrouver; le récit remonte généalogiquement jusqu'à Solon, puis il abandonne complètement le monde hellénique pour se retrouver dans le monde plus ancien de l'Égypte et, à travers ce registre égyptien, il débouche «au-delà de l'âge présent du cosmos, dans le cycle précédent ${ }^{4}$. Parallèlement à la remontée dans le temps vers les origines, on voit aussi le thème de la jeunesse et de l'ancienneté apparaître dans la descente de l'idée dans le présent - celui de Socrate, - où cette idée est encore vivante : «Le mythe égyptien de Critias est une confirmation de la polis socratique» ${ }^{43}$. La voie est libre pour que Timée développe alors une dimension future de la vérité, à savoir la vérité de l'âme, dans le mythe de la genèse de l'ordre du monde à partir de l'âme divine, car le Démiurge crée le temps physique comme une image mobile de l'éternité. L'ordre du monde et l'harmonie du mouvement céleste sont le produit de l'âme bien ordonnée du dieu. Par conséquent, l'harmonie de l'âme humaine est un microcosme de l'univers luimême, et la justice de la société bien ordonnée des hommes "socratiques" trouve sa place entre les deux autres incarnations de

\footnotetext{
41 E.Voegelin, Plato's Egyptian Myth, p. 311 (trad. J. Follon).

42 Ibid., p. 312.

43 Ibid., p. 315.
} 
l'Idée. Le mythe est plein d'espoir et d'encouragement pour la lutte des hommes, car, de même que le dieu a vaincu la résistance de la matière aveugle en la persuadant de suivre le mouvement ordonné de la raison (en grande partie avec succès), de même la raison qui est en nous, est invitée à accomplir sa tâche de gouvernante en compagnie du paradigme divin.

Dans son mythe égyptien, Platon établit un double rapport de l'idée avec le temps : un rapport mythique, tout d'abord, avec l'Athènes de l'âge cosmique actuel; un rapport concret, ensuite, mais à l'intérieur du mythe même, avec l'Égypte telle qu'elle est. Pourquoi considérait-il celle-ci, malgré ses imperfections, comme une incarnation de l'État idéal ? La réponse à cette question nous est suggérée par le mythe de l'Atlantide : même lorsque la Grèce fut menacée d'invasion, Athènes fut la seule à résister, parce que les cités étaient incapables de faire la guerre ensemble. En effet, elles n'étaient pas à même de se fédérer dans une union panhellénique, mais elles se trouvaient, au contraire, dans un état endémique de guerre de toutes contre toutes (l'expression apparait dans les Lois $^{44}$ ). Nous savons que Platon avait des ambitions politiques et que, dans sa jeunesse, il avait désespéré de sa cité natale, de cette Athènes qui s'était rendue coupable de la mort de Socrate et qui, à son époque, n'avait plus aucune foi collective dans la divine mesure de toute chose. Je suis convaincu qu'à la suite de cette déception, causée par sa propre cité et par les Grecs en général, son esprit fut attiré par la contemplation de cette Égypte qui avait réussi à maintenir l'unité et la puissance d'une constitution et de tout un peuple sur une période de temps d'une longueur incalculable. Et je crois que les faits nous autorisent à expliquer, comme Platon lui-même, l'impressionnante stabilité et l'interminable survivance de l'Égypte ancienne par sa «religion» ou, pour le dire en termes plus platoniciens, par l'harmonie et la consonance de ses lois (nomoi) et de sa culture avec la nature, et, à travers celle-ci, avec la puissance divine. Il y a un passage des Lois qui, à mes yeux, confirme cette hypothèse. Les trois hommes âgés qui, dans ce dialogue, réfléchissent ensemble sur les lois régissant les fêtes, l'éducation, la musique et la théologie d'une vraie société, sont d'accord pour condamner la culture des cités grecques de leur époque : à des degrés divers, en effet, celles-ci courent après la nouveauté et la licence dans les formes artistiques et la culture populaire, et elles ont perdu tout respect pour l'art sacré. Mais en Égypte, ce n'est nullement le cas :

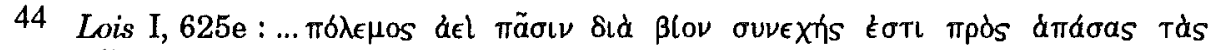

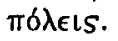


Clinias : De quelle façon dis-tu que la question a été en Égypte réglée législativement?

L'ATHÉNIEN : Rien que de l'apprendre, on est émerveillé ! Il y a fort longtemps en effet, à ce qu'il semble, qu'a été reconnue dans cette nation la thèse que j'expose présentement : à savoir que c'est à de belles attitudes et à de beaux chants que doit s'adonner la jeunesse de l'État dans sa pratique habituelle. Or, une fois qu'on eut déterminé ces attitudes et ces chants, on fit connaître dans les temples quels ils étaient et de quelle nature; et il n'était permis, ni aux peintres, ni à aucun de ceux dont c'est par ailleurs le métier de produire des attitudes ou encore quoi que ce fût d'analogue, de s'écarter de ces modèles en ouvrant de nouvelles voies, pas même d'imaginer rien qui différait des représentations traditionnelles. Á cette heure, c'est une chose qui n'est pas davantage permise, ni dans ces représenta tions figurées, ni dans la musique en son ensemble. Du reste, à examiner les peintures et les sculptures qui ont été faites dans ce pays il y a dix mille ans (et, quand je parle de dix mille ans, ce n'est pas une façon de dire, mais bien ce qui en est réellement), on se rendra compte que, comparées à celles qui sont l'œuvre des artistes d'à présent, elles ne sont en rien ni plus belles, ni plus laides, mais qu'elles attestent la même technique.

CLINIAS : C'est merveilleux, ce que tu dis là !

L'ATHÉNIEN : Bien mieux, cela dépasse tout sous le rapport de la législation et de l'art poétique : non qu'il soit impossible, là même, de découvrir d'autres dispositions qui ne valent pas cher! Toujours est-il que celles qui ont trait à la musique sont vraies et méritent d'être prises en considération : à savoir qu'il était décidément possible en pareille matière de légiférer avec une solide confiance sur les chants qui offrent une naturelle rectitude. Or, ce serait l'œuvre d'une divinité ou bien de quelque personnage divin, de la façon dont on dit là-bas que les mélodies qui se sont conservées pendant ce long laps de temps ont été l'œuvre de la déesse Isis. Par conséquent, c'est tout juste ce que je disais, s'il se trouvait quelqu'un qui fût capable de mettre la main sur ce qui est, de quelque façon que ce soit, la rectitude en cette matière, il devrait avec confiance en faire une loi et le prescrire : attendu que la recherche de ce qui nous donne le plaisir et nous épargne la peine, recherche qui consiste à chercher toujours la nouveauté en musique, ne possède peut-être pas une grande puissance pour ce qui est de ruiner, en l'accusant d'être vieille, la pratique chorale qui est consacrée : du moins en a-t-elle été là-bas complètement incapable, et même ç'a été tout le contraire 45 .

45 Lois II, 656d - 657 b (trad. Robin). On peut comparer avec Lois VII, 799a-b, où la discussion porte sur le besoin de conserver et de continuer les vertus de la bonne société même dans la musique, qui est l'expression extérieure des dispositions des hommes bons ou mauvais. 
Ainsi, le conservatisme de l'Égypte mérite d'être loué parce qu'il réalise et maintient avec la nature un accord qui est juste et qui permet à une vie supérieure de fleurir avec la bénédiction des dieux ${ }^{46}$.

\section{Le philosophe-roi et le pharaon}

Nous conclurons avec une question qui évoque une possibilité intéressante, mais qui reste du domaine de la conjecture. On pourrait s'étonner que Platon, qui savait tellement de choses sur l'Égypte et l'admirait beaucoup, ne dise rien de cette figure que les Égyptiens euxmêmes regardaient comme un dieu, à cause de l'ordre juste qui, à l'image du soleil, rayonnait de son trône sur tout le pays : le pharaon. Car il y a bien, dans l'œuvre de Platon, une figure royale, qui représente l'idéal d'un gouvernement divin, exercé par celui qui unit dans sa personne le pouvoir et l'amour de la sagesse, et cet idéal était au cœur de l'espérance de notre philosophe déjà à l'époque de ses voyages à Syracuse, c'est-à-dire bien avant qu'il n'en investisse la figure du philosophe-roi dans la République. Dès lors, ma question est de savoir si, dans l'âme socratique qui est amoureuse du Bien (soleil du monde intelligible), dans le dirigeant de la République qui est amoureux de la sagesse, dans ce roi, si peu grec, grâce auquel le soleil de l'Idée répand la vraie justice dans le monde temporel, nous n'avons pas finalement une figure inspirée par le paradigme du gouvernement pharaonique ${ }^{47}$.

46 C'était surtout le caractère sacré et religieux de l'art égyptien qui fascinait Platon. Les formes bi-dimensionnelles stylisées de l'art graphique lui semblaient écarter le risque d'illusion inhérent aussi bien à la vue qu'au style naturaliste de la Grèce de son temps, où les figures revêtaient l'apparence de la réalité. Les conventions graphiques bi-dimensionnelles soulignaient les proportions numériques exactes sans exposer aux tentations de l'illusionnisme "photographique». Peut-être ce qui attirait Platon dans l'art de l'Égypte était la même chose que ce qu'il admirait dans la dimension sacrée de la culture supérieure de ce pays, à savoir une échappée - dans l'ordre de la vie sociale et dans l'expérience artistique - du monde des phénomènes vers la réalité d'un univers moral transcendant et stable. On trouvera des réflexions à ce sujet dans H. SchaEFer, Principles of Egyptian Art, trad. et éd. par J. Baines, Oxford, 1974, p. 270-271. Pour une discussion récente et complète, voir W.M. D AVIES, Plato on Egyptian Art, in Journal of Egyptian Archaeology, 65 (1979), p. 121-127.

47 Rappelons ici qu'Hécatée d'Abdère devait développer une réflexion admirative sur la royauté pharaonique, incarnée par Ptolémée I (vers 300 av. J.-C.), et sur les qualités idéales des gouvernants. Son idéalisation de Ptolémée comme monarque constitutionnel bienfaisant et respectueux des lois s'écarte assez de 
Voegelin avance une hypothèse séduisante, qui suggère que l'esprit politique de Platon a pu l'orienter dans la direction des conceptions proches-orientales des rois-sauveurs, alors qu'il était à la recherche d'un paradigme pour sauver la Grèce menacée ${ }^{48}$ :

L'évolution de la conception platonicienne de l'ordre, jusqu'à la position défendue dans les Lois, doit être comprise dans le contexte de la politique grecque. Les cités n'avaient jamais trouvé le moyen de s'unifier à l'échelle nationale et territoriale, même quand la menace de la puissance perse était claire pour tout le monde. Le besoin d'une organisation politique plus large devait être, à cette époque, tellement évident, que la vision platonicienne d'un empire hellénique n'avait en principe rien d'extraordinaire. De fait, elle était si proche du courant de la politique pragmatique, qu'elle anticipait nettement la solution qui fut donnée au problème durant la génération suivante, avec la fondation de l'empire d'Alexandre, puis des monarchies hellénistiques. Et l'évocation par Platon du philosophe-roi n'est, sous un aspect, rien d'autre que l'expression de la recherche d'une figure hellénique correspondant aux rois sauveurs et aux pharaons des empires du Proche-Orient. Cette conception est même si peu extraordinaire qu'il est difficile d'imaginer comment un penseur politique dans cette situation aurait pu exiger moins que n'a fait Platon.

Il y a, heureusement, une remarque de Platon lui-même qui nous permet de considérer cette hypothèse de Voegelin comme quelque chose d'un peu plus substantiel qu'une pure vue de l'esprit. Dans le Politique, l'Étranger montre que la constitution qui est vraiment la meilleure, n'est pas une constitution écrite, ni non plus la constitution d'une oligarchie ou d'une démocratie, mais bien celle d'une royauté, soigneusement dotée des qualités requises pour incarner l'Idée. Une des qualifications de l'art royal est d'une importance particulière dans le présent contexte :

L'ÉTRANGER : Approchons-nous donc plus près encore de ceux que jusqu'à présent nous n'avons point soumis à la question. Or, il y a des gens, qui, dans l'ordre de l'art divinatoire, possèdent une portion d'une connaissance relative à un office : effectivement, on les considère, je pense, comme les interprètes auprès des hommes de la volonté des dieux.

LE JEUNE SOCRATE : OUi.

L'ÉTRANGER : $\AA$ son tour, c'est bien certain, la gent des prêtres, à en croire ce qu'affirme la coutume, est celle qui s'y connaît pour que, au moyen de

la perspective de Platon, mais une certaine influence de ce dernier sur le premier est fort plausible : voir O. MURRAY, Hecataeus of Abdera and Pharaonic Kingship, in Journal of Egyptian Archaeology, 56 (1970), p. 141 171.

48 E. Voegelin, Plato and Aristotle, p. 223 (trad. J. Follon). 
sacrifices, soient offertes aux dieux par nous autres hommes des offrandes qui leur agréent et pour que, dans nos prières, nous leur demandions de nous accorder la possession de téls ou tels biens. Or, ce sont là, je pense, d'un côté comme de l'autre, des parties d'un art relatif à un office.

LE JEUNE SOCRATE : Tout au moins est-ce ce que l'on constate!

L'ÉTRANGER : Et maintenant, nous voilà désormais, si je ne me trompe, comme au contact d'une trace conduisant à ce qui est le but de notre marche. C'est un fait, justement, que l'attitude des prêtres comme celle des devins se gonfle d'un prodigieux orgueil et que la grandeur des tâches auxquelles ils se livrent leur vaut une si respectueuse considération que, en Égypte, il n'est pas permis au roi de gouverner sans être pourvu de la prêtrise, et que, si d'aventure, issu d'une autre classe, il a commencé par user de violence pour s'emparer du pouvoir, il est plus tard forcé de se faire recevoir dans la classe en question. En outre, chez les Grecs également, en mainte contrée, on trouverait assigné aux plus importantes magistratures le privilège de célébrer les plus importants des sacrifices, en rapport à un tel objet; votre propre exemple, chacun le sait, ne confirme pas moins la vérité de ce que je dis, puisque, dit-on, dans ce pays-ci c'est au magistrat auquel le sort a donné le titre de Roi qu'a été dévolue la charge de célébrer ceux des antiques sacrifices qui sont les plus augustes et les plus essentiellement nationaux 49 .

Il est clair que "le magistrat auquel le sort a donné le titre de Roi» doit être compris comme une référence au second des archontes d'Athènes annuellement élus. L'argument de Platon est d'ordre historique et revient à dire qu'en Grèce, l'association de la royauté avec le sacrifice était on ne peut plus ancienne, mais aussi que, dans l'Athènes de son temps, il ne restait plus grand-chose de cette association. En généralisant l'argument, son esprit se tourne tout à fait spontanément vers l'Égypte, où la royauté sacrée, c'est-à-dire l'association ancienne entre la souveraineté royale et la prêtrise, avait été plus nettement préservée. La pertinence de ce passage par rapport à l'argumentation générale de mon exposé est claire, car il ne fait aucun doute que Platon était très sensible au caractère sacral de la royauté égyptienne. Cependant, même si nous prenons pleinement en compte cette sensibilité explicite de Platon, il n'y a, je l'avoue, aucun argument décisif qui montre que Platon ait eu à l'esprit les prêtres-rois d'Égypte quand il développa l'idéal d'une royauté vraiment sage. Mais on perçoit mieux ce que cette hypothèse a de plausible si l'on prend en considération l'indignation féroce de Platon contre Athènes et la Grèce, son souci

49 Polit. 290c-e (trad. Robin). Les mots grecs traduits dans l'éd. Robin par «les plus augustes et les plus essentiellement nationaux" sont : $\tau d \quad \sigma \epsilon \mu \nu \delta ́ \tau \alpha \tau \alpha ~ k a l$

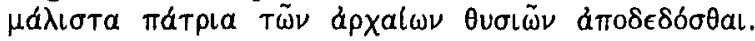


obsessionnel pour un gouvernement juste, l'admiration qu'il ressentait pour l'Égypte, sa compréhension étonnante de l'antique principe ordonnateur de cette nation, et, ce qui est peut-être le plus important, ce mythe de l'univers du Timée, dans lequel culmine la vision philosophique de sa période de maturité.

Centre De Wulf-Mansion

James McEVOY

Collège Thomas More

Chemin d'Aristote, 1

B - 1348 LouvaIN-LA-N EUVE 